\title{
CHARACTERIZATIONS OF TAUBERIAN OPERATORS AND OTHER SEMIGROUPS OF OPERATORS
}

\author{
M. GONZALEZ AND V. M. ONIEVA
}

(Communicated by John B. Conway)

\begin{abstract}
In this paper we present three characterizations of Tauberian operators in terms of: perturbations by compact operators, products with other operators, and restrictions to subspaces. We obtain also analogous characterizations for co-Tauberian operators and for other semigroups of operators related with the Tauberian and co-Tauberian ones.
\end{abstract}

\section{INTRODUCTION}

Tauberian operators, studied by Kalton and Wilansky [9], appear in different situations: summability [3], factorization of operators [1, 11], preservation of isomorphic properties of Banach spaces [11], equivalence between RadonNikodym property and Krein-Milman property [15], and generalized Fredholm operators [17, 18]. Recently Neidinger and Rosenthal [12] have obtained characterizations of Tauberian operators in terms of the closedness of images of closed sets.

In this paper we present characterizations of Tauberian operators and of other semigroups of operators studied in [4,5], formally analogous in some sense to the class of Tauberian operators. We prove that an operator $T \in L(X, Y)$ is Tauberian if and only if for every compact operator $K \in L(X, Y)$ the kernel $N(T+K)$ is reflexive; or for every Banach space $Z$ and $A \in L(Z, X)$, $T A$ weakly compact implies $A$ weakly compact; of for every subspace $M$ of $X$, if the restriction $T i_{M}$ is compact, then $M$ is reflexive. For co-Tauberian operators, i.e. operators whose conjugate is Tauberian [17], we give dual characterizations: the cokernel $Y / \overline{R(T+K)}$ is reflexive for every compact operator $K$; or for every Banach space $Z$ and $B \in L(Y, Z), B T$ weakly compact implies $B$ weakly compact; or for every subspace $N$ of $Y$, if $q_{N}$ is the quotient map onto $Y / N$ and $q_{N} T$ is compact, then $Y / N$ is reflexive.

Received by the editors February 7, 1989.

1980 Mathematics Subject Classification (1985 Revision). Primary 47B05, 47B30.

Key words and phrases. Tauberian operator, compact perturbation, ideal semigroup.

Supported in part by CAICYT grant $0185 / 85$. 
We also give analogous characterizations for the other semigroups, whose definitions are given below, and prove that the operators belonging to the semigroups preserve some isomorphic properties of Banach spaces in a similar manner as Tauberian operators.

For an operator ideal $V$, the space ideal $\operatorname{Sp}(V)$ is the class of all Banach spaces $X$ whose identity $I_{X}$ is in $V$, the dual operator ideal $V^{d}$ is the class of operators $K$ such that $K^{*}$ is in $V$, and $\operatorname{Sp}\left(V^{d}\right)=\left\{X / X^{*} \in \operatorname{Sp}(V)\right\}$ [13].

Let $U$ be one of the operator ideals $\mathrm{Co}$, WCo, Ro, CC, and WCC of all compact, weakly compact, Rosenthal, completely continuous, and weakly completely continuous operators, respectively. Note that $\operatorname{Sp}(U)$ is, respectively, the class of all finite-dimensional, reflexive, without copies of $l_{1}$, Schur or weakly sequentially complete Banach spaces.

In [4] and [5] we define two semigroups $\mathrm{SU}_{+}$and $\mathrm{SU}_{-}$of operators (whose definitions will be given below) such that $\mathrm{SCo}_{+}$and $\mathrm{SCo}_{-}$coincide with the classes $\mathrm{SF}_{+}$and $\mathrm{SF}_{-}$of upper and lower semi-Fredholm operators and they are contained in $\mathrm{SU}_{+}$and $\mathrm{SU}_{-}$, respectively. Also $\mathrm{SWCo}_{+}$coincides with the class of all Tauberian operators $[9,12]$, and $\mathrm{SCC}_{+}$with the class of all operators preserving mere Cauchy sequences considered in [10]. Moreover for $T$ in $\mathrm{SU}_{+}$ $\left(S U_{-}\right)$we have $N(T) \in \operatorname{Sp}(U) \quad\left(Y / \overline{R(T)} \in \operatorname{Sp}\left(U^{d}\right)\right)$, and $T+K$ is in $\mathrm{SU}_{+}$ $\left(S U_{-}\right)$for every operator $K$ in $U\left(U^{d}\right)$. In this way $\mathrm{SU}_{+}$and $\mathrm{SU}_{-}$can be seen as classes of "generalized" semi-Fredholm operators, with the operator ideals $U$ and $U^{d}$ acting as perturbation classes and the corresponding space ideals replacing the finite-dimensional spaces of classical Fredholm theory.

Given Banach spaces $X, Y, L(X, Y)$ will denote the set of all (continuous linear) operators from $X$ into $Y$; and $T^{*} \in L\left(Y^{*}, X^{*}\right)$ the conjugate operator of $T \in L(X, Y)$.

Recall that $T$ belongs to Co (resp., WCo, Ro) if $T$ maps bounded sequences onto sequences which have a convergent (resp., weakly convergent, weak Cauchy) subsequence.

$T$ belongs to CC (resp., WCC) if $T$ maps weakly Cauchy sequences onto convergent (resp., weakly convergent) subsequences.

$T$ belongs to $\mathrm{SCo}_{+}$(resp., $\mathrm{SWCo}_{+}, \mathrm{SRo}_{+}$) if every bounded sequence $\left(x_{n}\right)$ in $X$ such that $\left(T x_{n}\right)$ is convergent (resp., weakly convergent, weak Cauchy) has a convergent (resp., weakly convergent, weak Cauchy) subsequence.

$T$ belongs to $\mathrm{SCC}_{+}\left(\mathrm{SWCC}_{+}\right)$if every weak Cauchy sequence $\left(x_{n}\right)$ in $X$ such that $\left(T x_{n}\right)$ converges (weakly converges) is convergent (weakly convergent).

For $U \in\{\mathrm{Co}, \mathrm{WCo}, \mathrm{Ro}, \mathrm{CC}, \mathrm{WCC}\}, T$ belongs to $\mathrm{SU}_{-}$if the conjugate operator $T^{*} \in L\left(Y^{*}, X^{*}\right)$ belongs to $\mathrm{SU}_{+}$.

\section{The MAIN RESUlts}

A remarkable characterization of semi-Fredholm operators (see $[7 ; 6.12 .4]$ ) says that $T \in L(X, Y)$ is upper (lower) semi-Fredholm if and only if for each 
compact operator $K$ the kernel $N(T+K)$ (cokernel $Y / \overline{R(T+K)}$ ) is finitedimensional. In the following theorem we obtain an extension to the case of the semigroups $\mathrm{SU}_{+}$and $\mathrm{SU}_{-}$. Recall that $\mathrm{SWCo}_{+}$and $\mathrm{SWCo}_{-}$are the classes of all Tauberian and co-Tauberian operators, respectively; $\mathrm{WCo}=\mathrm{WCo}^{d}$ (Gantmacher theorem $[13 ; 4.4 .7])$ and the space ideal $\mathrm{Sp}(\mathrm{WCo})$ is the class of all reflexive spaces.

Theorem 1. Suppose $U \in\{\mathrm{Co}, \mathrm{WCo}, \mathrm{CC}, \mathrm{Ro}, \mathrm{WCC}\}$ and $T \in L(X, Y)$.

(a) $T$ is in $\mathrm{SU}_{+}$if and only if $N(T+K)$ belongs to $\mathrm{Sp}(U)$ for every compact operator $K$.

(b) $T$ is in $\mathrm{SU}_{-}$if and only if $Y / \overline{R(T+K)}$ belongs to $\mathrm{Sp}\left(U^{d}\right)$ for every compact operator $K$.

Proof. (a) If $T \in \mathrm{SU}_{+}$then we have that $N(T+K) \in \mathrm{Sp}(U)$ for every $K \in U$ [4; Theorem 1]. Since the compact operators are included in $U$, the direct implication is clear. For the converse we consider separately each operator ideal $U$.

The case $U=\mathrm{Co}$ is the result for upper semi-Fredholm operators mentioned above.

In the remaining cases, if the operator does not belong to $\mathrm{SU}_{+}$, we shall obtain a basic sequence $\left(x_{n}\right)$ in $X$ which is bounded and bounded away from 0 (i.e. $0<a \leq\left\|x_{n}\right\| \leq b$ for some real numbers $\left.a, b\right)$ and $\left\|T x_{n}-y\right\|$ converges to 0 for some $y \in Y$. The associated sequence of coefficient functionals of $\left(x_{n}\right)$ is bounded. Taking a bounded sequence $\left(f_{n}\right)$ in $X^{*}$ of extensions of these functionals and passing (if necessary) to a subsequence, we can suppose $\left\|T x_{n}-y\right\|\left\|f_{n}\right\|<2^{-n}$; then $A x:=\sum f_{n}(x)\left(T x_{n}-y\right)$ defines a compact operator. Moreover $(T-A) x_{n}=y$ for every $n$, and so $(T-A)$ is a one-dimensional operator in the subspace generated by $\left(x_{n}\right)$, which can be extended to a one-dimensional operator $L$ from $X$ into $Y$. Then $K:=A+L$ is compact and $N(T-K)$ contains the subspace generated by $\left(x_{n}\right)$, and this will show that $N(T-K)$ does not belong to $\operatorname{Sp}(U)$.

Case $U=$ WCo. Suppose $T$ is not Tauberian, i.e. $T$ does not belong to $\mathrm{SWCo}_{+}$. Then there exists a nonrelatively weakly compact, bounded set $A$ in $X$ such that $T(A)$ is relatively compact [9; Theorem 3.2], and by the Eberlein Smulian theorem we can find a bounded sequence $\left(x_{n}\right)$ in $A$ without weakly convergent subsequences such that $\left(T x_{n}\right)$ converges to $y \in Y$. By a result of Kadec and Pelczynski (see [16; p. 53]) $\left(x_{n}\right)$ has a basic subsequence, which we also denote with $\left(x_{n}\right)$, and $\left\|T x_{n}-y\right\|$ converges to zero. Since $\left(x_{n}\right)$ has no weakly convergent subsequences we conclude that $N(T-K)$ is not reflexive.

Case $U=\mathrm{CC}$. Suppose $T$ is not in $\mathrm{SCC}_{+}$. Then there exists a weakly convergent sequence $\left(x_{n}\right)$ in $X$ without convergent subsequences, such that $\left(T x_{n}\right)$ is convergent. Let $x$ be the weak limit of $\left(x_{n}\right)$. Clearly $\left(T x_{n}\right)$ converges to $T x$. Then $y_{n}:=x_{n}-x$ defines a weakly null and bounded away from zero 
sequence, such that $\left(T y_{n}\right)$ converges to 0 . Now, by using the Bessaga Pelczynski selection principle (see [2; p. 42]), we can find a basic subsequence of $\left(y_{n}\right)$ and construct a compact operator $K$ such that $N(T-K)$ is not Schur because $\left(y_{n}\right)$ is weakly convergent but not convergent.

Case $U=$ Ro. If $T$ is not in $\mathrm{SRo}_{+}$, then we can find a bounded sequence $\left(x_{n}\right)$ in $X$ without weakly Cauchy subsequences such that $\left(T x_{n}\right)$ is weakly Cauchy.

By Rosenthal characterization of Banach spaces containing no copies of $l_{1}$ [14], we can suppose $\left(x_{n}\right)$ equivalent to the $l_{1}$ basis. Then $y_{n}:=x_{2 n}-x_{2 n-1}$ is also equivalent to the $l_{1}$ basis and $\left(T y_{n}\right)$ converges weakly to 0 . Now we can find a sequence of integer numbers $i_{1}<\cdots<i_{n}<\cdots$ and a sequence of convex combinations $z_{n}:=a_{i_{n}+1} y_{i_{n}+1}+\cdots+a_{i_{n+1}} x_{i_{n+1}}$ such that $\left(T z_{n}\right)$ converges (in norm) to 0 ; since $\left(z_{n}\right)$ is still equivalent to the $l_{1}$ basis, $N(T-K)$ contains a subspace isomorphic to $l_{1}$.

Case $U=$ WCC. If $T$ is not in $\mathrm{SWCC}_{+}$, then there exists a weakly Cauchy sequence $\left(x_{n}\right)$ in $X$ without weakly convergent subsequences such that $\left(T x_{n}\right)$ converges weakly to some $y \in Y$. As in the case $U=$ Ro we construct a sequence of convex combinations $\left(z_{n}\right)$ of $\left(x_{n}\right)$ such that $\left(z_{n}\right)$ is weakly Cauchy without weakly convergent subsequences and $\left(T z_{n}-y\right)$ converges to 0 . Hence $N(T-K)$ is not weakly sequentially complete.

(b) If $T \in \mathrm{SU}_{-}$then $Y / \overline{R(T+K)}$ is in $\operatorname{Sp}(U)$ for every operator $K$ in $U$ [5; Proposition 2]. Hence, as in (a), the direct implication is clear. Note also that the result for $U=\mathrm{Co}$ is the above mentioned characterization of lower semi-Fredholm operators.

For the converse recall that $U / \overline{R(T)}$ is in $\operatorname{Sp}\left(U^{d}\right)$ if and only if its dual space $N\left(T^{*}\right)$ is in $\operatorname{Sp}(U)$. Suppose $T$ does not belong to $\mathrm{SU}_{-}$; then $T^{*}$ does not belong to $\mathrm{SU}_{+}$. We shall show that, applying to $T^{*}$ the method followed in (a), we can obtain a conjugate compact operator $K=L^{*}$.

We shall use the following result of Johnson and Rosenthal [8; Remark 3.1]: Given a sequence $\left(f_{n}\right)$ in $Y^{*}$ with 0 a weak ${ }^{*}$ cluster point of $\left(f_{n}\right)$ and $0<\lim \sup \left\|f_{n}\right\|<\infty$, then $\left(f_{n}\right)$ has a basic subsequence $\left(g_{m}\right)$ such that if $\left(F_{m}\right)$ is the (bounded) sequence of coefficient functionals of $\left(g_{m}\right)$ in the dual of the space $M$ generated by $\left(g_{m}\right)$ and $I$ from $Y$ into $M^{*}$ is defined by $(I x)(f)=f(x)$ for all $f \in M$ and $x \in Y$, then $I Y$ includes the closed subspace generated by $\left(F_{m}\right)$. In particular we can find a bounded sequence $\left(x_{m}\right)$ in $Y$ such that $g_{i}\left(x_{j}\right)=\delta_{i j}$.

Now if $\left(h_{n}\right)$ is a bounded and bounded away from 0 basic sequence in $Y^{*}$ such that $\left\|T^{*} h_{n}-g\right\|$ converges to 0 for some $g \in X^{*}$, then $\left(h_{n}\right)$ has a weak ${ }^{*}$ cluster point $h$ and $T^{*} h=g$ since the unit ball of $Y^{*}$ is weak ${ }^{*}$ compact. Let $f_{n}:=h_{n}-h$. We observe that $\left(f_{n}\right)$ has the same subsequence properties as $\left(h_{n}\right)$ in each case. Applying the result of Johnson and Rosenthal we obtain a basic subsequence $\left(g_{m}\right)$ for which there exists a biorthogonal sequence $\left(x_{n}\right)$ in $Y$; then the compact operator $K$ defined by $K f:=\sum f\left(x_{m}\right) T^{*} g_{m}$ is the conjugate 
of the operator $L \in \operatorname{Co}(X, Y)$ given by $L x:=\sum g_{m}(T x) x_{m}$, and $N\left(T^{*}-K^{*}\right)$ includes the subspace generated by $\left(g_{n}\right)$. This shows that $N\left(T^{*}-K^{*}\right)$ does not belong to $\operatorname{Sp}(U)$; hence $Y / \overline{R(T+K)}$ is not in $\operatorname{Sp}\left(U^{d}\right)$.

Observation 1. It is an easy consequence of the definitions that the operator ideals considered in the paper are related as follows:

$$
\text { Co } \subset \mathrm{WCo} \cap \mathrm{CC}, \quad \mathrm{WCo} \subset \mathrm{Ro} \cap \mathrm{WCC}, \quad \mathrm{CC} \subset \mathrm{WCC} .
$$

From Theorem 1 it is immediate that the inclusions obtained changing the operator ideal $U$ by the corresponding semigroup $\mathrm{SU}_{+}$(or $\mathrm{SU}_{-}$) are true.

Next we present the algebraic characterizations of the semigroups. Given a (closed) subspace $M$ of $X, i_{M}$ will denote the inclusion of $M$ into $X$, and $q_{M}$ the quotient map onto $X / M$.

Theorem 2. Suppose $U \in\{\mathrm{Co}$, WCo, CC, Ro, WCC $\}$ and $T \in L(X, Y)$.

(a) $T$ belongs to $\mathrm{SU}_{+}$if and only if for every Banach space $Z$ and $A \in$ $L(Z, X)$ we have that $T A \in U$ implies $A \in U$, or equivalently for every subspace $M$ of $X, T i_{M} \in U$ implies $M \in \mathrm{Sp}(U)$.

(b) $T$ belongs to $\mathrm{SU}_{-}$if and only if for every Banach space $Z$ and $B \in$ $L(Y, Z)$ we have that $B T \in U^{d}$ implies $B \in U^{d}$, or equivalently for every subspace $N$ of $Y, q_{N} T \in U^{d}$ implies $Y / N \in \operatorname{Sp}\left(U^{d}\right)$.

Proof. (a) Case $U=$ WCo. If $T$ is Tauberian and $T A \in$ WCo, then for every bounded sequence $\left(z_{n}\right)$ in $Z$ the sequence $\left(T A z_{n}\right)$ has a weakly convergent subsequence. By the definition of Tauberian operator, $\left(A z_{n}\right)$ must have a weakly convergent subsequence; hence $A \in \mathrm{WCo}$. In particular for $A=i_{M}$ we obtain $i_{M} \in \mathrm{WCo}$, hence $M$ reflexive since the operator ideal WCo is injective [13].

Conversely, if $T i_{M} \in$ WCo implies $M$ reflexive, it is clear that the same is true for $T+K$ with $K \in \mathrm{Co}$; in particular $N(T+K)$ is reflexive for every $K \in \mathrm{Co}$, and then we derive from Theorem 1 that $T$ is Tauberian; i.e. $T \in \mathrm{SWCo}_{+}$.

In the other cases the proof is analogous. Note that all the operator ideals considered in the theorem are injective [13].

(b) Case $U=$ WCo. If $T \in \mathrm{SWCo}_{-}$and $B T \in \mathrm{WCo}^{d}=\mathrm{WCo}$, then $T^{*}$ is Tauberian and $T^{*} B^{*} \in \mathrm{WCo}$. As in (a) we have $B^{*} \in \mathrm{WCo}$, and so $B \in$ WCo as well. In particular, for $B=q_{N}$ we obtain $q_{N} \in$ WCo, hence $Y / N$ is reflexive since WCo is surjective [13]. Conversely, if $q_{N} T \in$ WCo implies $Y / N$ reflexive, then the same is true for $T+K$ for every compact operator $K$. So $Y / \overline{R(T+K)}$ is reflexive for every $K$, and from Theorem 1 we conclude $T \in \mathrm{SWCo}_{-}$. The other cases are analogous. Note that $U^{d}$ is surjective for all the operator ideals considered in this theorem [13].

Observation 2. In [5] we considered two other operator ideals defined in terms of the weak ${ }^{*}$ convergence in dual spaces. 
$T$ belongs to $\mathrm{Gr}$ : Grothendieck operators (resp., Cd : condensed operators) if $T^{*}$ maps weak ${ }^{*}$ convergent sequences onto weak convergent sequences (resp., sequences with weakly Cauchy subsequences). Note that $\mathrm{Sp}(\mathrm{Cd})$ and $\mathrm{Sp}(\mathrm{Gr})$ are the classes of all Banach spaces with no quotient isomorphic to $c_{0}$ [6; Theorem 2.1] and Grothendieck Banach spaces, respectively.

The proof of part (b) in the above theorems can be adapted to the semigroups SGr_ and SCd defined as follows: $T$ belongs to $S G r_{-}$(resp., SCd_) if every $w^{*}$-convergent sequence $\left(f_{n}\right)$ in $Y^{*}$ such that $\left(T^{*} f_{n}\right)$ is $w$-convergent (resp., $w$-Cauchy), has a $w$-convergent (resp., $w$-Cauchy) subsequence. Note that for these operator ideals the definition of $\mathrm{SU}_{+}$given for the other semigroups has no sense.

We obtain for $T \in L(X, Y)$ and $U \in\{\mathrm{Gr}, \mathrm{Cd}\}$ that the following conditions are equivalent:

(1) $T \in \mathrm{SU}_{-}$.

(2) $Y / R(T+K) \in \operatorname{Sp}(U)$ for every $K \in \operatorname{Co}(X, Y)$.

(3) For every Banach space $Z$ and $B \in L(Y, Z), B T \in U$ implies $B \in U$.

(4) For every subspace $N$ of $Y, q_{N} T \in U$ implies $Y / N \in \operatorname{Sp}(U)$.

A remarkable property of Tauberian operators is the preservation of some isomorphic properties of Banach spaces: If there exists a Tauberian operator $T \in L(X, Y)$ and $Y$ is reflexive, does not contain $l_{1}$ or $c_{0}$, or is weakly sequentially complete, then $X$ has the same property (for these and other related results see $\left[11 ;\right.$ p. 94]). We shall show that the semigroups $\mathrm{SU}_{+}$and $\mathrm{SU}_{-}$also preserve some isomorphic properties.

Proposition 1. Let $T \in L(X, Y)$.

(a) If $T \in \mathrm{SU}_{+}$and $Y \in \mathrm{Sp}(U)$, then $X \in \mathrm{Sp}(U)$.

(b) If $T \in \mathrm{SU}_{-}$and $X \in \mathrm{Sp}\left(U^{d}\right)$, then $Y \in \mathrm{Sp}\left(U^{d}\right)$.

Proof. (a) If $Y \in \operatorname{Sp}(U)$ then $T=T I_{X} \in U$, and so $I_{X} \in U$, i.e. $X \in \operatorname{Sp}(U)$. The proof of $(b)$ is analogous.

\section{REFERENCES}

1. W. J. Davis, T. Figiel, W. B. Johnson and A. Pelczynski, Factoring weakly compact operators, J. Funct. Anal. 17 (1974), 311-327.

2. J. Diestel, Sequences and series in Banach spaces, Springer, New York, 1984.

3. D. J. H. Garling and A. Wilansky, On a summability theorem of Berg, Crawford and Whitley, Math. Proc. Cambridge Philos. Soc. 71 (1972), 495-497.

4. M. Gonzalez and V. M. Onieva, Semi-Fredholm operators and semigroups associated with some classical operator ideals, Proc. Roy. Irish Acad. Sect. A 88 (1988), 35-38.

5. __ Semi-Fredholm operators and semigroups associated with some classical operator ideals II, Proc. Roy. Irish. Acad. Sect. A 88 (1988), 119-124.

6. _ Lifting results for sequences in Banach spaces, Math. Proc. Cambridge Philos. Soc. 105 (1989), 117-121.

7. R. Harte, Invertibility and singularity for bounded linear operators, Marcel Dekker, New York, 1988. 
8. W. B. Johnson and H. P. Rosenthal, On $w^{*}$ basic sequences and their application to the study of Banach spaces, Studia Math. 43 (1972), 77-92.

9. N. J. Kalton and A. Wilansky, Tauberian operators in Banach spaces, Proc. Amer. Math. Soc. 57 (1976), 251-255.

10. D. H. Martin and J. Swart, A characterization of semi-Fredholm operators defined on almost reflexive Banach spaces, Proc. Roy. Irish Acad. Sect. A 86 (1986), 91-93.

11. R. D. Neidinger, Properties of Tauberian operators on Banach spaces, Ph.D. Thesis, University of Texas, 1984.

12. R. D. Neidinger and H. P. Rosenthal, Norm-attainment of linear functionals on subspaces and characterizations of Tauberian operators, Pacific J. Math. 118 (1985), 215-228.

13. A. Pietsch, Operator ideals, North-Holland, Amsterdam, 1980.

14. H. P. Rosenthal, A characterization of Banach spaces containing $l_{1}$, Proc. Nat. Acad. Sci. U.S.A. 71 (1974), 2411-2413.

15. W. Schachermayer, For a Banach space isomorphic to its square the Radon-Nikodym property and the Krein-Milman property are equivalent, Studia Math. 81 (1985), 329-339.

16. I. Singer, Bases in Banach spaces II, Springer, New York, 1981.

17. D. G. Tacon, Generalized semi-Fredholm transformations, J. Austral. Math. Soc. A34 (1983), 60-70.

18. K. W. Yang, The generalized Fredholm operators, Trans. Amer. Math. Soc. 219 (1976), 313326.

Departamento de Matematicas, Facultad de Ciencias, 39005 Sautander, Spain

Departamento de Matematicas, Facultad de Ciencias, 50009 Zaragoza, Spain 